\title{
APPLICATION OF A SELF-TUNING REGULATOR TO A SOLAR POWER PLANT
}

\author{
F. R. Rubio*, E. F. Camacho* and R. Carmona**

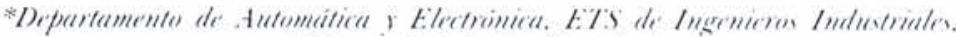

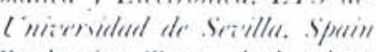

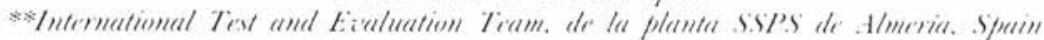

\begin{abstract}
This paper presents an application of a self-tuning regulator to the distributed collector field of a solar power plant. The distributed collector field consists of a series of parabolic mirrors that reflect solar radiation on a pipe where oil gets heated while circulating. The purpose of the regulator is to maintain the oil out let temperature as near as possible to a desired level. This is accomplished by varying the flow of the fluid through the field. The field exhibits a variable delay time that depends on the control variable (flow). The transfer function of the process varies with factors such as irradiance level, mirrors reflectance and oil inlet temperature. The self-tuning regulator uses an identifier with a variable forgetting factor and an adaptive PI controller. The paper also describes the heuristic used to make the regulator work an the experiences cobtained at the SSPS solar
\end{abstract}

Keywords. Adaptive control; Digital control; Energy control; Power control; Self-adjusting systems.

\section{INTRODUCTION}

A lot of works showing the advantages of adaptive controllers (Astrom 1983, Landau 1974 ) have appeared in the Literature 1974) have appeared in the Literature
since self-tuning regulators were first introduced by Astrom and Wittenmark (1973). Most of these works are theoretical and in spite of the fact that adaptive control has shown advantages when applied to a variety of processes (Borisson 1976, Buchholf 1979, Kallstrom 1979, Narendra 1980, Dumont 1982, Rubio 1982, etc.) it is not yet widely accepted at industrial level. This paper presents an application of an adaptive regulator to control the outlet temperature of the ACUREX distributed collector field of the SSPS plant of Tabernas.

The objective of the control system in the distributed collector field is to maintain the outlet oil temperature at a desired level in spite of disturbances such as changes in the solar, irradiance level (caused by clouds or the time of day mirrors reflectivity and inlet oil temperature.

The distributed collectors fields is a nonlinear system which can be approximated by a linear system when considering small disturbances. In the design of any regulator the operating point of the plant must be born in mind. However in a solar energy plant the operating point varies according to the time of day or disturbances caused by clouds, and therefore it is not possible to design a fixed regulator fully guaranteed to work. Because of this self-tuning regulator seems to be a good solution to this problem.

The plant to be controlled is described in section 2 of this article. Section 3 gives a description of the control algorithm used, section 4 comments upon the results obtained and section 5 is dedicated to the conclusions.

\section{A DESCRIPTION OF THE PLANT}

The system to which the self-tuning control referred to in this article has been applied is that formed by the distributed collectors field (ACUREX) of the solar energy plant of Tabernas.

The distributed collectors field consists mainly of a pipe line through which oil is flowing and on to which the suns rays are concentrated by means of parabolic mirrors in order to heat the oil. It consists of 480 modules arranged in twenty lines which form ten parallel loops as shown in $\mathrm{fig}$. 1. The field is also provided a sun seeking mechanism which causes the mirrors to revolve around an axis parallel to that of the pipe line.

On passing through the field the oil is heated and then introduced into a storage tank to be used for the generation of electrical energy. The cold inlet oil to 


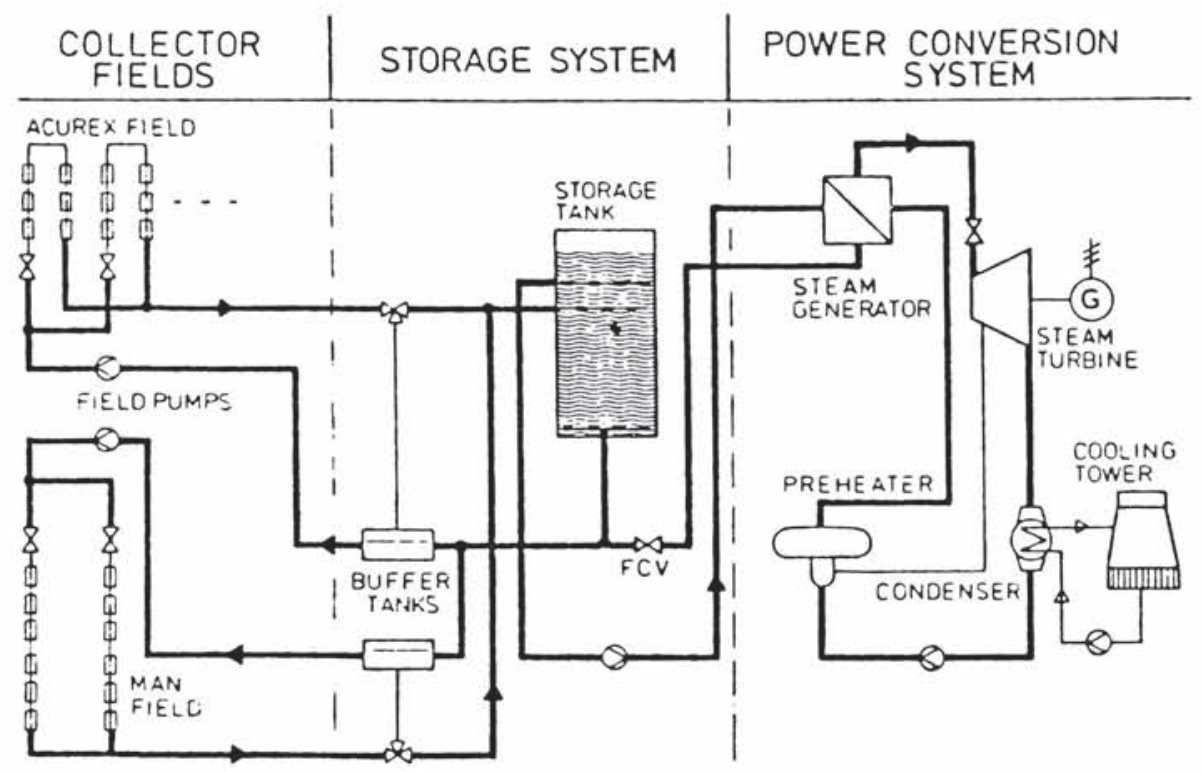

FIG. 1 SIMPLIFIED DCS PROCESS FLOW DIAGRAM

the field is extracted from the bottom of the storage tank.

The system is provided with a three way valve which allows the oil to be recycled in the field until the its outlet in the field until the its outlet storage tank. A more detailed description of the field can be found in (Kalt 1982).

The temperature in the field can be given by the following equations:

$$
\begin{aligned}
& P_{m} C_{m} A_{m} \frac{\hat{\partial} T_{m}}{\partial t}=I n_{0} D-H_{l} G\left(T_{m}-T_{a}\right) \\
&-L_{t}\left(T_{m}-T_{f}\right) \\
& P_{f} C_{f} A_{f}-\frac{\partial T_{f}}{\partial t}+P_{f} C_{f} \dot{V} \frac{\partial T_{f}}{x} \\
&=L H_{t}\left(T_{m}-T_{f}\right)
\end{aligned}
$$

where the subindex $m$ refers to the metal and $f$ to the fluid and:

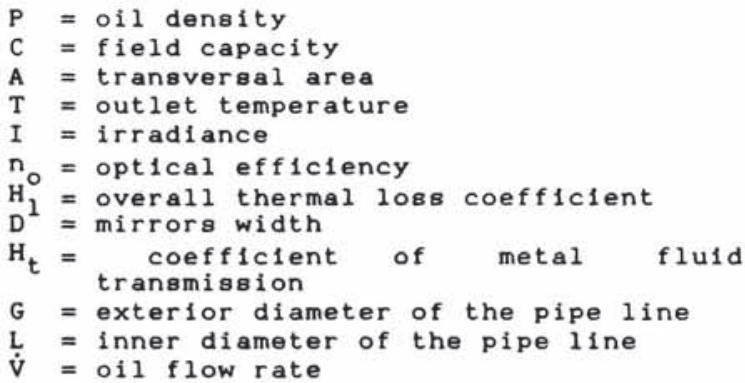

These equations are only applicable to the active zones of the field. That is, those parts of the pipe line where solar radiations are collected. Parts of the field, passive zones, exists where it is not possible to collect solar energy due to geometrical conditions as is the case of the joints between the modules. These zones constitute a considerable part of the field and they are characterized by having nil irradiance and different loss constants.

The above equations were used to simulate the system in a computer dividing one of the loops into a 100 pieces and using a model of concentrated parameters for each piece. The model was contrasted against the real data obtained from the field. The parameter of the model were adjusted so that it reproduced the behaviour of the system (Camacho 1977).

The outlet temperature of the sixth loop is used to control the field as if acts as the pilot loop, in this way the total outlet temperature is maintained within an acceptable range.

\section{THE CONTROL ALGORITHM}

Because of the variability of the plants characteristics as has been previously mentioned an adaptive control algorithm has been chosen.

The adaptive control structure used corresponds to that of a self-tuning regulator (STR) ( $\mathrm{fig} .2$ ), which, in brief consists in calculating the parameters of the regulator supposing that the fields parameters are those given by means of an identification algorithm. In this case recursive least square a identification algorithm has been used.

In each sampling period the self-tuning regulator consists of the following steps:

1) An estimation of the parameters of a linear model by measuring the inlet and outlet values of the process.

2) The adjustment of the parameters of the regulator.

3) The calculation of the control signal.

4) The supervision of the correct working of the control. 
The regulator is composed of two parts (fig.3), a P.I. in the feedback loop and a feedforward regulator calculated using the available information on the field.

The system model

We presume that the system can be modelled as a stable process, invariant in time and that can be linealized with just one input and one output, so that it can be described by the following linear difference equation:

$y(k)+a_{1} y(k-1)+\ldots+a_{n} y(k-n)=b_{1}$

$u(k-d-1)+b_{2} u(k-d-2)+b_{n} u(k-d-n)+v(k)$

and also by the vectorial form:

$$
y(k)=x^{T}(k) p+v(k)
$$

where :

$$
\begin{aligned}
& x^{T}(k)=\left(\begin{array}{l}
-y(k-1),-y(k-2), \ldots-y(k-n) \\
u(k-d-1), u(k-d-2), \ldots u(k-d-n))
\end{array}\right. \\
& p^{T}=\left(a_{1}, a_{2}, \ldots a_{n}, b_{1}, b_{2}, \ldots b_{n}\right) \\
& u(k)=U(k)-\text { Uoo } \\
& y(k)=Y(k)-Y \text { ref }
\end{aligned}
$$

$U(k)$ and $Y(k)$ are the input and output values of the system at the instant $k$, Uoo is the average value of the input signal, Yoo is the value of the reference and $v(k)$ is a noise signal statistically independent and stationary of zero mean. The $z$ transfer function of this system can be written as:

$y(z)=\frac{B\left(z^{-1}\right)}{A\left(z^{-1}\right)} z^{-d} u(z)+\frac{1}{A\left(z^{-1}\right)} v(z)$

where,

$A\left(z^{-1}\right)=1+a_{1} z^{-1}+a_{2} z^{-2}+\ldots+a_{n} z^{-n}$ $B\left(z^{-1}\right)=b_{1} z^{-1}+b_{2} z^{-2}+\ldots+b n z^{-n}$ The first quotient $B\left(z^{-1}\right) / A\left(z^{-1}\right)$ represents the model of the field, and the second $\left.1 / \mathrm{A}^{-1}\right)$ represents the model of the disturbances.

Feedforward Control.

In a concentrated representation of the plant the internal energy variation of the field can be given by:

$C \frac{d T}{d t}=N_{0} R G_{n}-V P C_{p}\left(T-T_{i}\right)-H_{1}\left(T_{m}-T_{a}\right)$

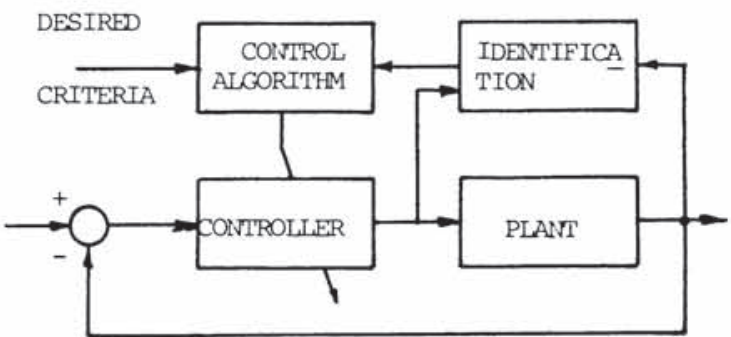

Fig. 2 Self-tuning regulator

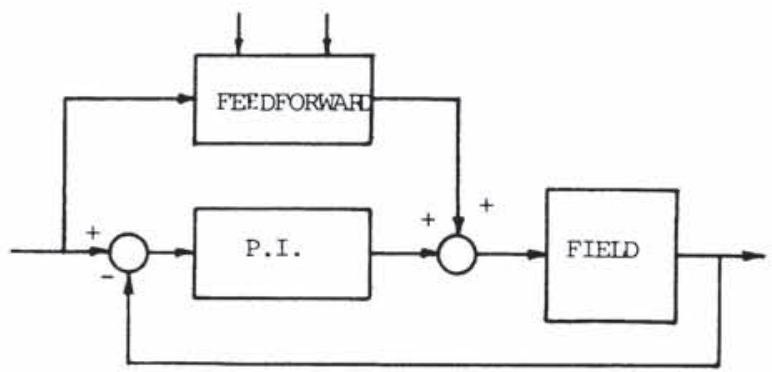

Fig. 3 Basic control layout.

In the permanent regime we have that:

$$
V=\frac{N_{0} R G_{n}-H_{1}\left(T_{m}-T_{a}\right)}{P C_{p}\left(T_{r}-T_{i}\right)}
$$

This last equation can be approximated as:

$$
V=\frac{K_{1} R G_{n}-K_{2}}{T_{r}-T_{i}}
$$

where,

$\mathrm{T}_{\mathrm{r}}=$ Reference temperature.

$\mathrm{T}^{\mathrm{r}}=$ Inlet tempersture.

$\mathrm{G}_{\mathrm{n}}=$ Irradiance.

This equation gives the flow as a function of the reference temperature, inlet temperature, and the irradiance. The constants $K_{1}$ and $K_{2}$, have been determined experimentafly, having the values of $\mathrm{K}_{1}=$ 0.93 and $K_{2}=155$ (Carmona, 1983).

\section{Feedback control}

Using a closed loop regulator is necessary to compensate for any error which may be produced in the flow calculation by the feedforward controller. Given that this flow is a good estimation of the permanent regime flow, the value of the control signal becomes satured at $+20 \%$ of the open loop value, with this, greater stability is obtained in the transients, as the regulation band is reduced.

The plant under consideration presents a long delay (between 2 and 8 minutes) that varies with the inlet flow. For this type of system where the delay is much greater than the order of the system, the controller described below can be used (Isermann, 1981).

Supposing that the plant is modelled using the equation $y(k)=b u(k-d)$, where $d$ is the delay in sampling periods and $b$ the system gain, the minimum settling time controller can be expressed by:

$$
u(k)=u(k-d)+e(k) / b
$$

If instead of directly using this controller we approximate a P.I. regulator so that it has the same behaviour, we obtain:

$u(k)=u(k-1)+q_{0} e(k)+q_{i} e(k-1)$

where:

$$
\begin{aligned}
& q_{0}=1 /(2 b) ; \\
& q_{i}=-q_{0}(d-2) / d ;
\end{aligned}
$$




\begin{abstract}
It can be shown that this regulator is less sensitive to the error in the accuracy of the delay (Isermann, 1981). and therefore it is more advisable to use it rather than the controller (1), given the characteristics of the process. Therefore a P.I. has been used in the feedback loop. It should also be remembered that when an error occurs in the determination of the delay it is better to consider it as greater than the estimated, in order to ensure the stability.
\end{abstract}

\section{Identification of Parameter Algorithm}

The parameters identifier is a very important part of the self-tuning controllers. It should be recursive, as if must work in real time with the process and therefore it will a greatly influence the minimum sampling time we can obtain for the control system. In the same way, the stability of the system depends largely on the estimator convergence. There are various types of identifiers which are dealt with in the relative literature. Generally, the most used methods is the recursive least squares are, because of its simplicity and its good convergence characteristics. The algorithm is performed by the following steps:

1. Select the initial values of $P(k)$ and $\hat{p}(k)$.

2. Read the new values of $y(k+1)$ and $u(k+1)$.

3. Calculate the a priori error: $e(k+1)=y(k+1)-x^{T}(k+1) \hat{p}(k)$

4. Calculate $\mathrm{L}(\mathrm{k}+1)$ given by the expression:

$$
L(k+1)=\frac{P(k) X(k+1)}{c(k)+x^{T}(k+1) P(k) x(k+1)}
$$

5. Calculate the new parameter estimated given by:

$$
\hat{p}(k+1)=\hat{p}(k)+L(k+1) e(k+1)
$$

6. Actualize the covariance matrix.

$$
P(k+1)=\left(I-L(k+1) x^{T}(k+1)\right) \frac{P(k)}{c(k)}
$$

7. Calculate the new forgetting factor $c(k+1)$.

$$
c(k+1)=1-\left(1-x^{T}(k+1) L(k+1)\right) \frac{e(k+1)^{2}}{\text { So }}
$$$$
\text { If } c(k+1)<c_{\text {min }} \text { Then } c(k+1)=c_{\text {min }}
$$

8. Actualize the measurements vector $x(k+2)$.

9. Make $k=k+1$ and return to step 2 .

\section{Sampling and delay time}

The sampling time used has been set to ten seconds. This time is reasonable in respect to the time constant of the system, being in the range:

$$
(1 / 15) \mathrm{T}_{95}<\mathrm{T}_{\mathrm{m}}<(1 / 4) \mathrm{T}_{95}
$$

T95: Time taken to reach $95 \%$ of the
signal. $T_{m}$ : Sampling time.

The parameter (d) in the model under consideration is an integer which represents the delay of the system. In this process it is variable with the flow, and can be calculated as a function of the physical dimensions of the plant and of the flow, resulting that:

$$
d=\frac{80+187.5 * 5.3}{\dot{V} \star T_{m}}
$$

The previous values of the control signal are stored in a vector, in which they shift. Because the delay is variable the first idea was to shift the information within this vector at a constant rate and to take as the output the component of this vector corresponding to the delay. This method causes problems in the vector in which the input enter in one end, and go out of the other varying their shifting rate according to the delay was used. This method reproduces what really happens in the system because an element of fluid is displaced along the tube at a speed which varies according to the flow at a given moment. In this way satisfactory behaviour of the identifier has been achieved.

\section{Forgetting factor}

Given the fact that the plant is a continuously being identified as a linear system, the parameter of the linear model vary with time. In order to reduce the vary with time. In order to reduce the forgetting factor (c) (Fortescou 1981), as has been described previously. Notice that for $c=1$ we have the least squares identifier with infinite memory and the matrix $P(k)$ decreases monotonously so the estimator gain can reach zero. As the system must follow the parameter variations a forgetting factor (c<1) must be used. On the other hand if the working point does not change, the product $P(k) X(k)$ can be zero and therefore $P(k+1)=P(k) / c$. If $c<1, P(k)$ can grow greatly, making the identifier very sensitive to any change. To avoid this a variable forgetting factor is used. This factor is made to equal $I$ if the trace of $P(k)$ is greater than a certain value. At the same time, the trace of $P(k)$ is not allowed to go below a prefixed value by adding a matrix $R$ to $P(k+1)$.

\section{Supervision.}

A basic requirement for the adaptive control algorithm to be stable is that the estimated parameters converge on certain values. Certain conditions are necessary in order to guarantee this convergence, and these depend on the type of disturbances and whether the input is persistently exciting.

A great improvement in the behaviour of the closed loop system can be achieved in many cases by using a level of supervision which can check things such as:

- The estimated parameters. 
- The control variable.

- The evolution of the identifier.

In the concrete case of the solar energy plant the following steps are taken:

a) With regards to the plant identification we know that the system gain is negative and inferior to a certain value. Because of this the identified gain is filtered before being considered valid, in order to avoid a mistaken identification. This can be produced in cases where the irradiance decreases suddenly. The identifier works using the variations in the temperature and flow with respect to the values of the permanent regime. These values are given by the reference temperature and by the flow calculated by the feedforward controller.

b) The trace of the covariance matrix of the identifier is continuously checked. taking the steps considered previously

c) The control variable is saturated at + $20 \%$ of the value of the flow calculated by the feedforward controller. Furthermore a cautions control is used in the beginning modifying the expression (2) as follows:

$q_{0}=1 /(2 \star b+\operatorname{trace}(p(k)) \star b)$

RESULTS.

Before applying the regulator to the process a series of tests were carried out by simulation in order to validate and proof its usefullness. These test were aimed at analysing the behaviour of the regulator when faced with changes the regulator when faced with changes in reference and all types of disturbances. Different working points have been considered and the system has been subjected to small changes of reference and small disturbances as well as sudden disturbances.

A model of distributed parameters has been used to simulate the process, which has been validated and identified with real data from the solar energy plant.

Fig. 4 shows the behaviour of the regulator when faced with a slow variation of the irradiance, keeping the temperature reference at 280 degrees. Fig. 5 shows the system reaction to reference changes of between 280 and 290 degrees behaving well after the first reference change, which belongs to the first phase of adaption.

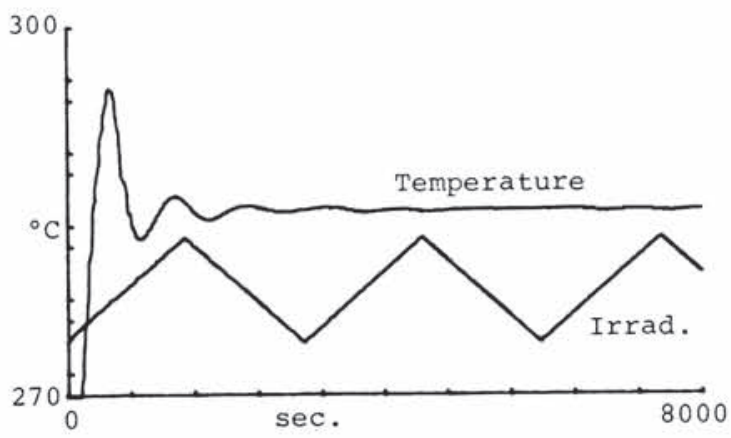

Figure 4. Irradiance changes

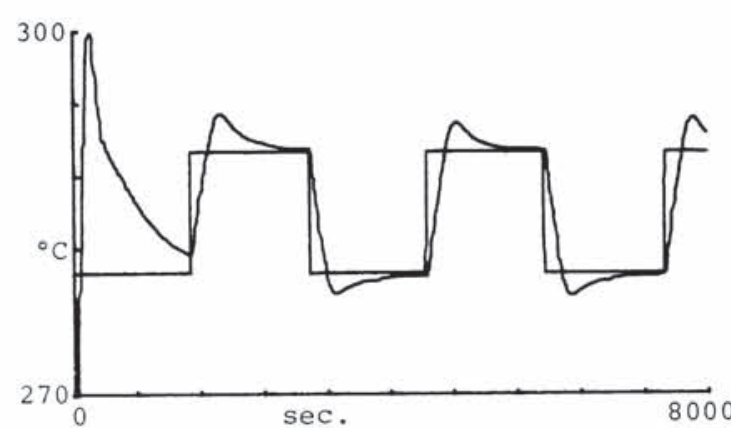

Figure 5 . Set point changes

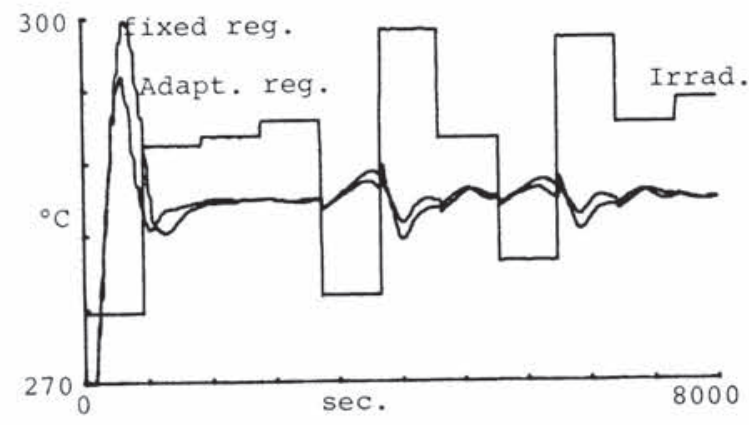

Figure 6 Regulators comp.

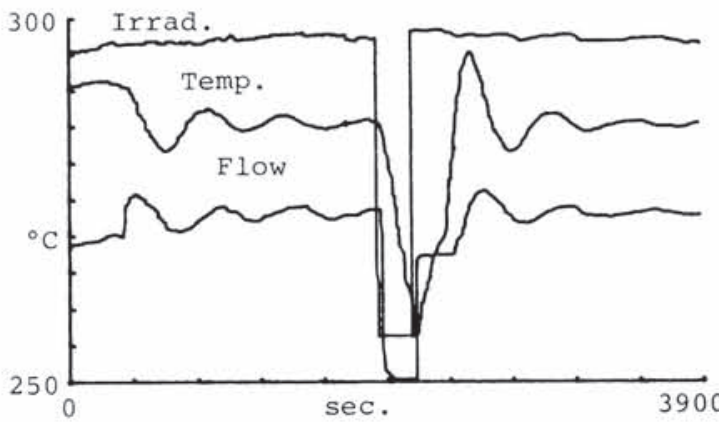

Figure 7 . Set point changes Cloud perturbation

Fig. 6 shows the behaviour of the self tuning regulator and of a fixed regulator when the irradiance change suddenly and randomly. It has been proved that the mean square error of the output in the case of the selftuning regulator is half that of the corresponding one of the fixed regulator.

Fig. 7 to 10 correspond to tests done in the SSPS plant of Almerla. Fig. 7 shows the outlet oil temperature when the reference is changed from 290 to 280 degrees, a slow variation in the irradiance and sharp change in this due to a small cloud passing, can be seen.

Fig. 8 corresponds to the starting-up phase of the process and regulator. The reference temperature was set at 280 degrees. The evolution of the identified parameter b can be seen in this figure. Notice that the outlet oil temperature has a small overshoot. 

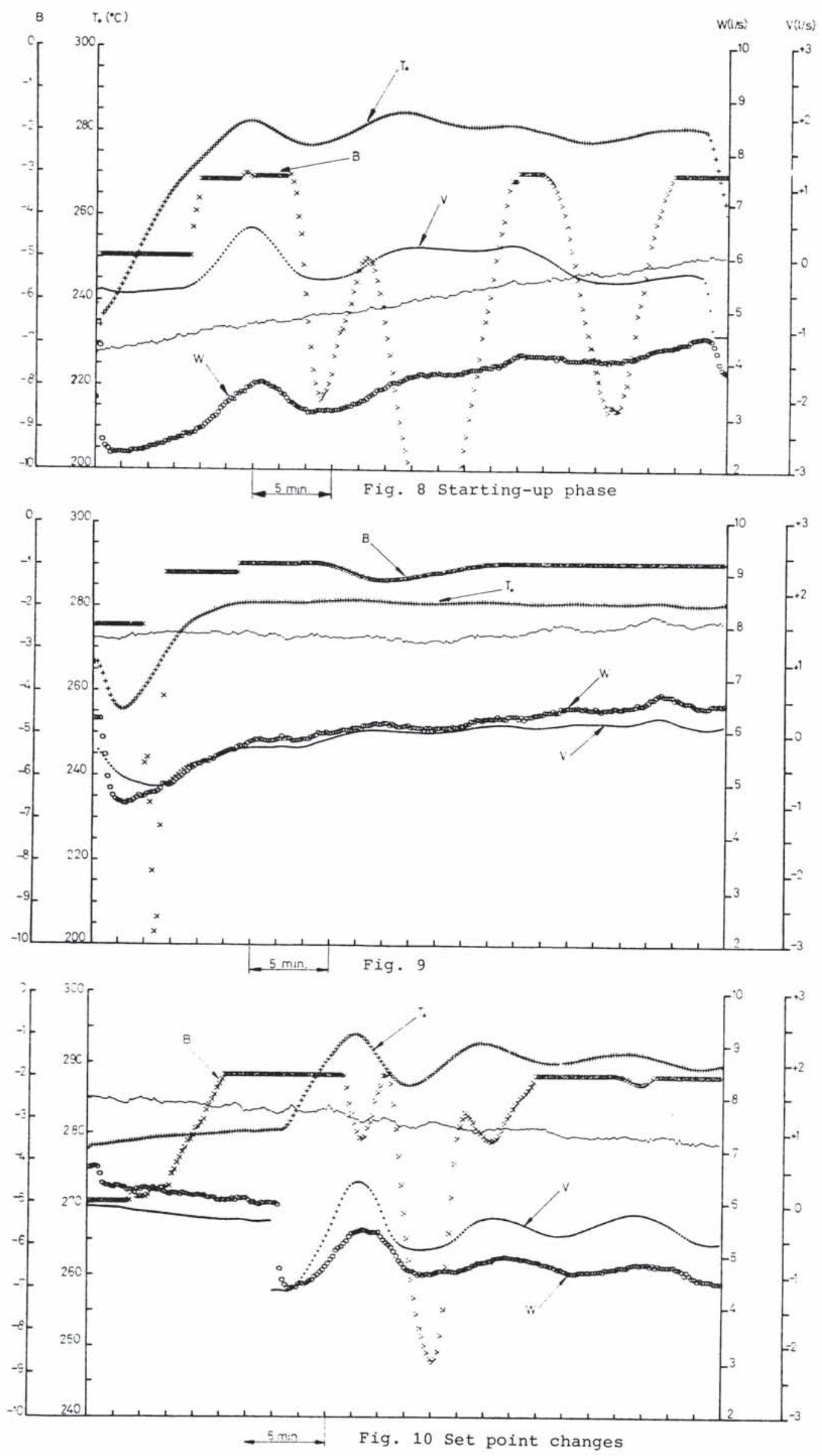
Fig. 9 shows the response of the plant when the oil inside the pipe line has been cool down by getting the mirrors out of focus. It can be seen that the outlet ofl temperature reaches the set point ( 280 degrees) satisfactorily.

The response of the system when the set point is changed from 280 to 290 degrees and the irradiance is decreasing slowly can be seen in fig. 10 .

\section{CONCLUSIONS}

An adaptive regulator to control the field of collectors in a solar energy plant is presented in this paper. This regulator has been successfully tried out under various working conditions in the ACUREX field of the solar energy plant SSPS of Almeria. In the same way other types of self-tuning regulators are being tested, one of which being the design of a regulator using the pole assignment method.

\section{REFERENCIAS}

Astrom, K.J. and Wittenmark, P.D. (1973) On Self-tuning Regulators, Automatica Vol 9 pp 185-199.

Astrom, K.J.

(1983),

Theory and Applications of Adaptive Control: A Survey, Automatica Vol 19-5 pp $471-486$

Borisson, U. and Syding, R. (1976), Self-tuning Control of an Ore Crusher. Automatica Vol 12 pp $1-7$.

Buchholt, F.
Self-tuning and $\begin{aligned} & \text { Kummel, } \\ & \text { Control }\end{aligned}$ of (1979),
PH-Neutralization Process, Automatica Vol 15 pp 665-671.

Camacho, E.F. (1977), Identificación de Sistemas no Lineales, Ajuste y Estimación de parámetros, Tes1s doctoral. ETSII Univ. de Sevilia.

Carmona, R. y Mart1n, J. (1983), Digital DCS Controls: The SSPS Experience First Term Workshop. Tabernas (Almeria).

Dumont, G.A. (1982), Self-tuning Control of a Chip Refiner Motor Load, Automatica Vol 18-3 pp 307-314.

Isermann, R. (1981), Digital Control Systems, Springer-verlag Berlin Heidelberg New York.

Kallstrom,C.G. et al. (1979), Adaptive Autopilots for Tankers, Automatica Vol 15 PP 241-254.

Kalt, A. et al. (1982), Distributed Collector System Plant Construction Report, IEA-SSPS Operating Agent DFVLR, Cologne, FRG.

Landau, I.D. (1974), A Survey of Model Reference Adaptive Techniques - Theory and Applications, Automatica Vol 10 pp 353-379.

Narendra, K.S. and Monopol1, R.V. (1980), Applications of Adaptive Control, Academic Press.

Rubio, F.R. et al. (1982), Studies for the Aplication and Adaptive Controller to Hidroturbine Generators, Proc. of the IFAC Software for Computer Control (Sococo), Madrid. 